\title{
A study on the anti-thermal $\mathrm{Dy}^{3+} / \mathrm{Eu}^{3+}$ co-doped $\mathrm{BaLa}_{4} \mathrm{Si}_{3} \mathrm{O}_{13}$ red phosphors for w-LEDs and optical thermometry applications
}

Fan $\mathrm{Liao}^{\dagger}$, Bingqing Shen ${ }^{\dagger}$, Weiwei $\mathrm{Wu}^{\dagger}$, Yuanpeng Zhang ${ }^{\star}$ and Jianxu $\mathrm{Hu}^{\dagger}$,*

${ }^{\dagger}$ Key laboratory of Photo-electronic Materials, Ningbo University, Ningbo, Zhejiang, 315211, China

Đak Ridge National Laboratory, 1 Bethel Valley Rd, Oak Ridge, TN 37830, USA

${ }^{*}$ Corresponding authors: Jianxu Hu (hujianxu@nbu.edu.cn)

\section{Supporting Information}

\section{Contents}

Table S1. Crystallographic data of $5 \mathrm{~mol}^{2} \mathrm{Dy}^{3+} / x$ mol $\% \mathrm{Eu}^{3+}$ co-doped $\mathrm{BaLa}_{4} \mathrm{Si}_{3} \mathrm{O}_{13}$ phosphors $(x=0,1,3,5,7,9$ and 11) from Rietveld refinement.

Table S2. Comparison of thermal stability for the reported $\mathrm{Eu}^{3+}$ and the synthesized $\mathrm{Dy}^{3+} / \mathrm{Eu}^{3+}$ co-doped $\mathrm{BaLa}_{4} \mathrm{Si}_{3} \mathrm{O}_{13}$ phosphors.

Table S3. Fitting parameters for fluorescence intensity ratio (FIR) as a function of temperature.

Table S4. Comparison of thermal stability for the reported $\mathrm{Eu}^{3+}$ and the synthesized $\mathrm{Dy}^{3+} / \mathrm{Eu}^{3+}$ co-doped $\mathrm{BaLa}_{4} \mathrm{Si}_{3} \mathrm{O}_{13}$ phosphors.

Figure S1. (a) Diffuse reflectance spectra of undoped $\mathrm{BaLa}_{4} \mathrm{Si}_{3} \mathrm{O}_{13}$ phosphor. (b) The dependence of $\left[F\left(R_{\infty}\right) h v\right]^{2}$ on the photon energy $h v$.

Figure S2. (a)-(f) Emission spectra of the synthesized $\mathrm{BaLa}_{4} \mathrm{Si}_{3} \mathrm{O}_{13}$ phosphors in a temperature range from 298 to $523 \mathrm{~K}$.

Figure S3. (a)-(d) The dependence of the integrated emission intensity at 480, $575 \mathrm{~nm}$ of $\mathrm{Dy}^{3+}$, and $616 \mathrm{~nm}$ of $\mathrm{Eu}^{3+}$ on temperature for $\mathrm{Dy}^{3+} / \mathrm{Eu}^{3+}$ co-doped $\mathrm{BaLa}_{4} \mathrm{Si}_{3} \mathrm{O}_{13}$ phosphors.

Figure S4. Decay curves monitored at (a) $480 \mathrm{~nm}$, and (b) at $575 \mathrm{~nm}$ in a temperature range from 298 to $523 \mathrm{~K}$ for the $5 \mathrm{~mol} \% \mathrm{Dy}^{3+} / 11 \mathrm{~mol} \% \mathrm{Eu}^{3+}$ co-doped $\mathrm{BaLa}_{4} \mathrm{Si}_{3} \mathrm{O}_{13}$ phosphors. The excitation wavelength is $350 \mathrm{~nm}$. 
References 
Table S1. Crystallographic data of $5 \mathrm{~mol} \% \mathrm{Dy}^{3+} / x$ mol $\% \mathrm{Eu}^{3+}$ co-doped $\mathrm{BaLa}_{4} \mathrm{Si}_{3} \mathrm{O}_{13}$ phosphors ( $x=0,1,3,5,7,9$ and 11) from Rietveld refinement.

\begin{tabular}{|c|c|c|c|c|c|c|c|}
\hline Sample & $5 \% \mathrm{Dy}^{3+}$ & $\begin{array}{c}5 \% \\
\mathrm{Dy}^{3+} / 1 \% \\
\mathrm{Eu}^{3+} \\
\end{array}$ & $\begin{array}{c}5 \% \\
\mathrm{Dy}^{3+} / 3 \% \\
\mathrm{Eu}^{3+} \\
\end{array}$ & $\begin{array}{c}5 \% \\
\mathrm{Dy}^{3+} / 5 \% \\
\mathrm{Eu}^{3+}\end{array}$ & $\begin{array}{c}5 \% \\
\mathrm{Dy}^{3+} / 7 \% \\
\mathrm{Eu}^{3+}\end{array}$ & $\begin{array}{c}5 \% \\
\mathrm{Dy}^{3+} / 9 \% \\
\mathrm{Eu}^{3+}\end{array}$ & $\begin{array}{c}5 \% \\
\mathrm{Dy}^{3+} / 11 \% \\
\mathrm{Eu}^{3+}\end{array}$ \\
\hline $\begin{array}{l}\text { Crystal } \\
\text { system }\end{array}$ & Hexagonal & Hexagonal & Hexagonal & Hexagonal & Hexagonal & Hexagonal & Hexagonal \\
\hline Space group & $\mathrm{P} 63 / \mathrm{m}$ & $\mathrm{P} 63 / \mathrm{m}$ & $\mathrm{P} 63 / \mathrm{m}$ & $\mathrm{P} 63 / \mathrm{m}$ & $\mathrm{P} 63 / \mathrm{m}$ & $\mathrm{P} 63 / \mathrm{m}$ & $\mathrm{P} 63 / \mathrm{m}$ \\
\hline$a(\AA)$ & 9.6554 & 9.6503 & 9.6469 & 9.6437 & 9.6388 & 9.6315 & 9.6264 \\
\hline$b(\AA)$ & 9.6554 & 9.6503 & 9.6469 & 9.6437 & 9.6388 & 9.6315 & 9.6264 \\
\hline$c(\AA)$ & 7.1558 & 7.1546 & 7.1532 & 7.1521 & 7.1410 & 7.1395 & 7.1366 \\
\hline$V\left(\AA^{3}\right)$ & 577.73 & 577.03 & 576.51 & 576.04 & 574.56 & 573.57 & 572.73 \\
\hline$\alpha(\mathrm{deg})$ & 90 & 90 & 90 & 90 & 90 & 90 & 90 \\
\hline$\beta(\operatorname{deg})$ & 90 & 90 & 90 & 90 & 90 & 90 & 90 \\
\hline$\gamma(\operatorname{deg})$ & 120 & 120 & 120 & 120 & 120 & 120 & 120 \\
\hline$\chi^{2}$ & 3.44 & 3.52 & 3.29 & 3.77 & 3.38 & 4.21 & 3.57 \\
\hline$R_{p}(\%)$ & 3.67 & 3.77 & 4.21 & 4.18 & 4.22 & 3.95 & 2.86 \\
\hline$R_{w p}(\%)$ & 5.52 & 5.29 & 5.09 & 5.43 & 5.29 & 5.13 & 4.27 \\
\hline
\end{tabular}


Table S2. Comparison of thermal stability for the reported $\mathrm{Eu}^{3+}$ and the synthesized $\mathrm{Dy}^{3+} / \mathrm{Eu}^{3+}$ co-doped $\mathrm{BaLa}_{4} \mathrm{Si}_{3} \mathrm{O}_{13}$ phosphors.

\begin{tabular}{|c|c|c|c|c|}
\hline Host & $\begin{array}{c}\text { Activated } \\
\text { ions }\end{array}$ & $\begin{array}{c}\text { Heating } \\
\text { temperature }\end{array}$ & $\begin{array}{l}\text { Residual } \\
\text { intensity }\end{array}$ & Literature \\
\hline $\mathrm{Sr}_{1.7} \mathrm{Zn}_{0.3} \mathrm{CeO}_{4}$ & $\mathrm{Eu}^{3+}$ & $423 \mathrm{~K}$ & $\sim 36 \%$ & 1 \\
\hline $\mathrm{MgY}_{4} \mathrm{Si}_{3} \mathrm{O}_{13}$ & $\mathrm{Eu}^{3+}$ & $423 \mathrm{~K}$ & $\sim 45 \%$ & 2 \\
\hline $\mathrm{LaBWO}_{6}$ & $\mathrm{Eu}^{3+}$ & $423 \mathrm{~K}$ & $\sim 57 \%$ & 3 \\
\hline $\mathrm{Na}_{3} \mathrm{Sc}_{2}(\mathrm{PO})_{4}$ & $\mathrm{Eu}^{3+}$ & $423 \mathrm{~K}$ & $\sim 73 \%$ & 4 \\
\hline $5 \% \mathrm{Dy} / 1 \% \mathrm{Eu} \mathrm{BaLa} 4 \mathrm{Si}_{3} \mathrm{O}_{13}$ & $\mathrm{Eu}^{3+}$ & $423 \mathrm{~K}$ & $\sim 85 \%$ & This work \\
\hline $5 \% \mathrm{Dy} / 3 \% \mathrm{Eu} \mathrm{BaLa} 4 \mathrm{Si}_{3} \mathrm{O}_{13}$ & $\mathrm{Eu}^{3+}$ & $423 \mathrm{~K}$ & $\sim 95 \%$ & This work \\
\hline $5 \% \mathrm{Dy} / 5 \% \mathrm{Eu} \mathrm{BaLa} \mathrm{Si}_{3} \mathrm{O}_{13}$ & $\mathrm{Eu}^{3+}$ & $423 \mathrm{~K}$ & $\sim 100 \%$ & This work \\
\hline $5 \% \mathrm{Dy} / 7 \% \mathrm{Eu} \mathrm{BaLa} 4 \mathrm{Si}_{3} \mathrm{O}_{13}$ & $\mathrm{Eu}^{3+}$ & $423 \mathrm{~K}$ & $\sim 102 \%$ & This work \\
\hline $5 \% \mathrm{Dy} / 9 \% \mathrm{Eu} \mathrm{BaLa} 4 \mathrm{Si}_{3} \mathrm{O}_{13}$ & $\mathrm{Eu}^{3+}$ & $423 \mathrm{~K}$ & $\sim 104 \%$ & This work \\
\hline $5 \% \mathrm{Dy} / 11 \% \mathrm{Eu} \mathrm{BaLa} 4 \mathrm{Si}_{3} \mathrm{O}_{13}$ & $\mathrm{Eu}^{3+}$ & $423 \mathrm{~K}$ & $\sim 108 \%$ & This work \\
\hline
\end{tabular}


Table S3. Fitting parameters for fluorescence intensity ratio (FIR) as a function of temperature.

\begin{tabular}{lcccc}
\hline Samples & $B$ & $C$ & $\Delta E$ & $R^{2}$ \\
\hline $5 \% \mathrm{Dy} / 5 \% \mathrm{Eu}$ & $0.85793 \pm$ & $58.26537 \pm$ & $0.13615 \pm$ & 0.98693 \\
& 0.19311 & 26.39419 & 0.02247 & \\
$5 \% \mathrm{Dy} / 7 \% \mathrm{Eu}$ & $1.31328 \pm$ & $802.53724 \pm$ & $0.23052 \pm$ & 0.9985 \\
& 0.05892 & 166.6286 & 0.00958 & \\
$5 \% \mathrm{Dy} / 9 \% \mathrm{Eu}$ & $1.81995 \pm$ & $16600.54594 \pm$ & $0.32976 \pm$ & 0.99934 \\
& 0.06508 & 2968.43018 & 0.00817 & \\
$5 \% \mathrm{Dy} / 11 \% \mathrm{Eu}$ & $2.12236 \pm$ & $81569.94459 \pm$ & $0.39714 \pm$ & 0.99276 \\
\hline
\end{tabular}


Table S4. Comparison of thermal stability for the reported $\mathrm{Eu}^{3+}$ and the synthesized $\mathrm{Dy}^{3+} / \mathrm{Eu}^{3+}$ co-doped $\mathrm{BaLa}_{4} \mathrm{Si}_{3} \mathrm{O}_{13}$ phosphors.

\begin{tabular}{|c|c|c|c|c|}
\hline Host & $\begin{array}{c}\text { Activated } \\
\text { ions }\end{array}$ & $\operatorname{Sr}\left(\% \mathrm{~K}^{-1}\right)$ & $T_{\max }(\mathrm{K})$ & Literature \\
\hline$\overline{\mathrm{MgAl}_{2} \mathrm{O}_{4}}$ & $\mathrm{Dy}^{3+} / \mathrm{Eu}^{3+}$ & 0.24 & 420 & 5 \\
\hline $\mathrm{KBaGd}\left(\mathrm{WO}_{4}\right)_{3}$ & $\mathrm{Dy}^{3+} / \mathrm{Eu}^{3+}$ & 0.64 & 458 & 6 \\
\hline $\mathrm{SrWO}_{4}$ & $\mathrm{Dy}^{3+} / \mathrm{Eu}^{3+}$ & 1.71 & 335 & 7 \\
\hline $\mathrm{Ba}_{5} \mathrm{Gd}_{8} \mathrm{Zn}_{4} \mathrm{O}_{21}$ & $\mathrm{Er}^{3+} / \mathrm{Yb}^{3+}$ & 0.36 & 200 & 8 \\
\hline$(\mathrm{Ca}, \mathrm{Sr})_{10} \mathrm{Li}\left(\mathrm{PO}_{4}\right)_{7}$ & $\mathrm{Ce}^{3+} / \mathrm{Mn}^{2+}$ & 0.40 & 473 & 9 \\
\hline $\mathrm{LaOBr}$ & $\mathrm{Ce}^{3+} / \mathrm{Tb}^{3+}$ & 0.42 & 433 & 10 \\
\hline $\mathrm{Yb}_{3} \mathrm{Al}_{5} \mathrm{O}_{12}$ & $\mathrm{Er}^{3+} / \mathrm{Mo}^{6+}$ & 0.48 & 467 & 11 \\
\hline $\mathrm{Ca} 8 \mathrm{ZnLa}\left(\mathrm{PO}_{4}\right)_{7}$ & $\mathrm{~Tb}^{3+} / \mathrm{Eu}^{3+}$ & 0.53 & 298 & 12 \\
\hline $\mathrm{NaYF}_{4}$ & $\mathrm{Ce}^{3+} / \mathrm{Tb}^{3+}$ & 0.65 & 563 & 13 \\
\hline $\mathrm{SrLu}_{2} \mathrm{O}_{4}$ & $\mathrm{Bi}^{3+} / \mathrm{Eu}^{3+}$ & 0.87 & 543 & 14 \\
\hline $5 \% \mathrm{Dy} / 5 \% \mathrm{Eu} \mathrm{BaLa} 4 \mathrm{Si}_{3} \mathrm{O}_{13}$ & $\mathrm{Dy}^{3+} / \mathrm{Eu}^{3+}$ & 0.56 & 380 & This work \\
\hline $5 \% \mathrm{Dy} / 7 \% \mathrm{Eu} \mathrm{BaLa} 4 \mathrm{Si}_{3} \mathrm{O}_{13}$ & $\mathrm{Dy}^{3+} / \mathrm{Eu}^{3+}$ & 0.82 & 462 & This work \\
\hline $5 \% \mathrm{Dy} / 9 \% \mathrm{Eu} \mathrm{BaLa} 4 \mathrm{Si}_{3} \mathrm{O}_{13}$ & $\mathrm{Dy}^{3+} / \mathrm{Eu}^{3+}$ & 1.26 & 476 & This work \\
\hline $5 \% \mathrm{Dy} / 11 \% \mathrm{Eu} \mathrm{BaLa} 4 \mathrm{Si}_{3} \mathrm{O}_{13}$ & $\mathrm{Dy}^{3+} / \mathrm{Eu}^{3+}$ & 1.46 & 497 & This work \\
\hline
\end{tabular}



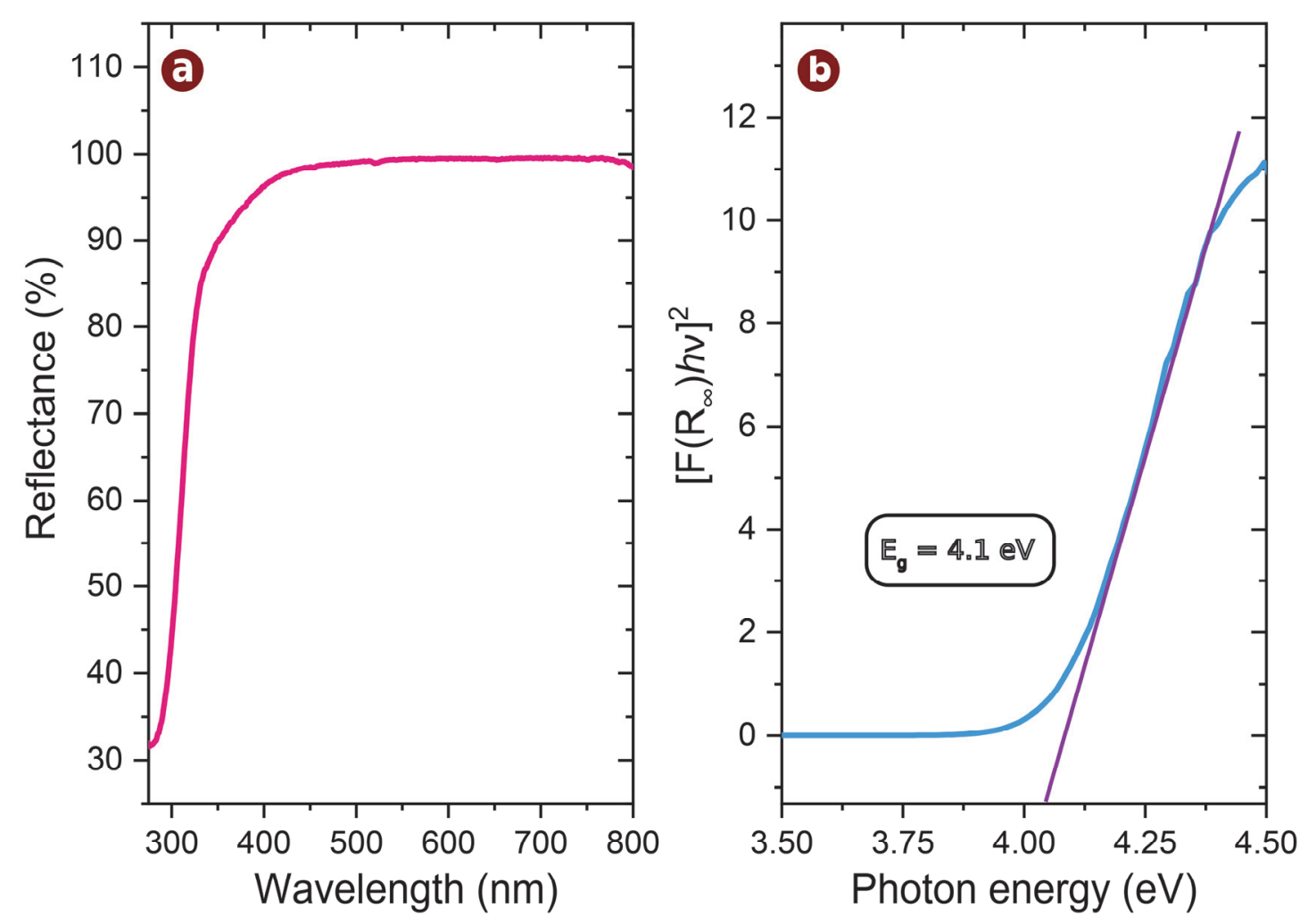

Figure S1. (a) Diffuse reflectance spectra of undoped $\mathrm{BaLa}_{4} \mathrm{Si}_{3} \mathrm{O}_{13}$ phosphor. (b) The dependence of $\left[F\left(R_{\infty}\right) h v\right]^{2}$ on the photon energy $h v$.

The energy gap $\left(\mathrm{E}_{\mathrm{g}}\right)$ of $\mathrm{BaLa}_{4} \mathrm{Si}_{3} \mathrm{O}_{13}$ phosphor can be evaluated by the following equation:

$\left[F\left(R_{\infty}\right) h v\right]^{n}=A\left(h v-E_{g}\right)$

Where $h v$ denotes photon energy, $A$ is a proportional constant, $n$ equals to 2 for direct allowed transition. $F\left(R_{\infty}\right)$ is the Kubelka-Munk function, which can be expressed as: $\left[F\left(R_{\infty}\right)\right]=(1-R)^{2} / 2 R$

Where $R$ is the reflectance coefficient. $\left[F\left(R_{\infty}\right) h v\right]^{2}$ is plotted against $h v$ according to the Tauc method, from which the energy bandgap is determined to be $\sim 4.1 \mathrm{eV}$. 

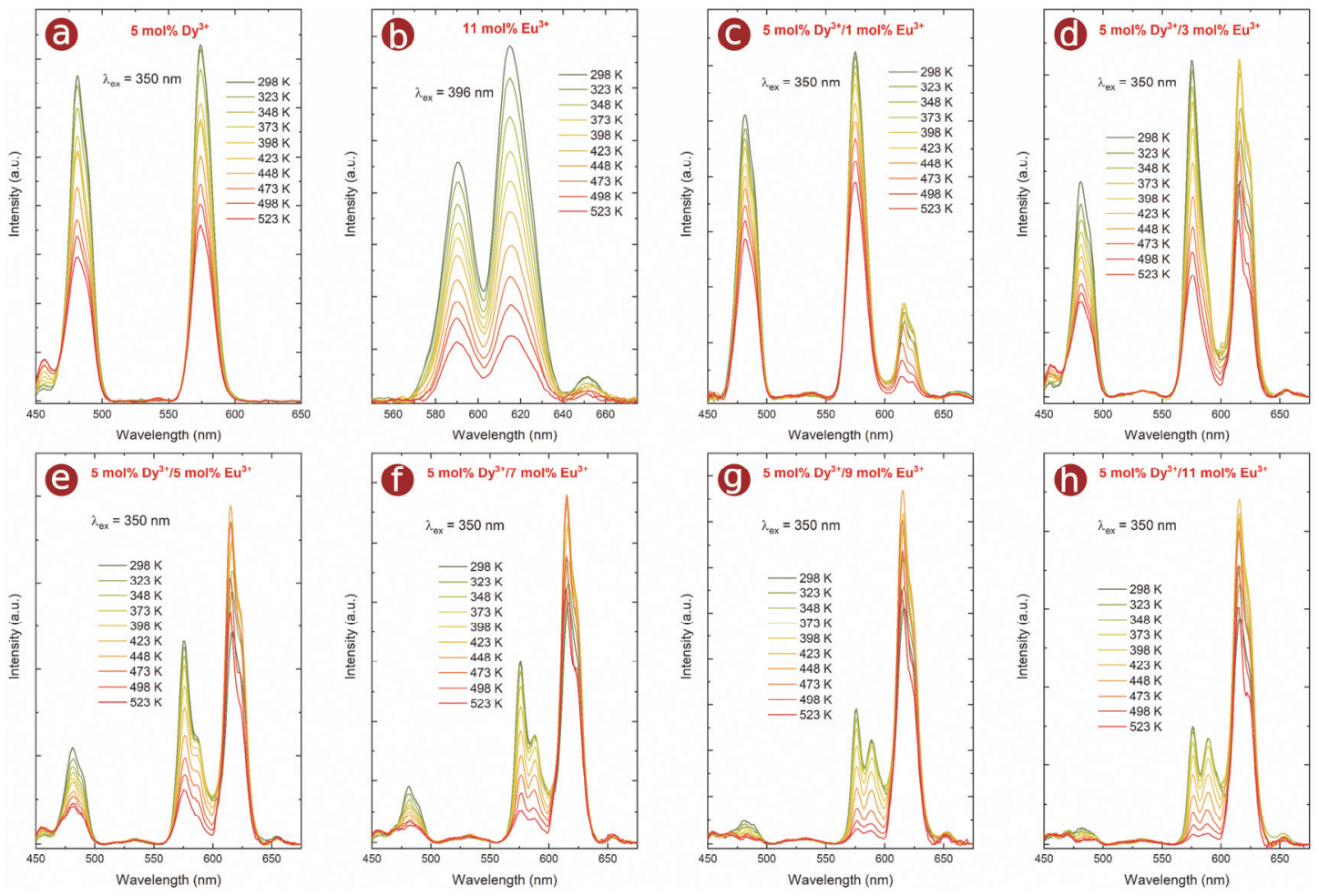

Figure S2. (a)-(f) Emission spectra of the synthesized $\mathrm{BaLa}_{4} \mathrm{Si}_{3} \mathrm{O}_{13}$ phosphors in a temperature range from 298 to $523 \mathrm{~K}$. 

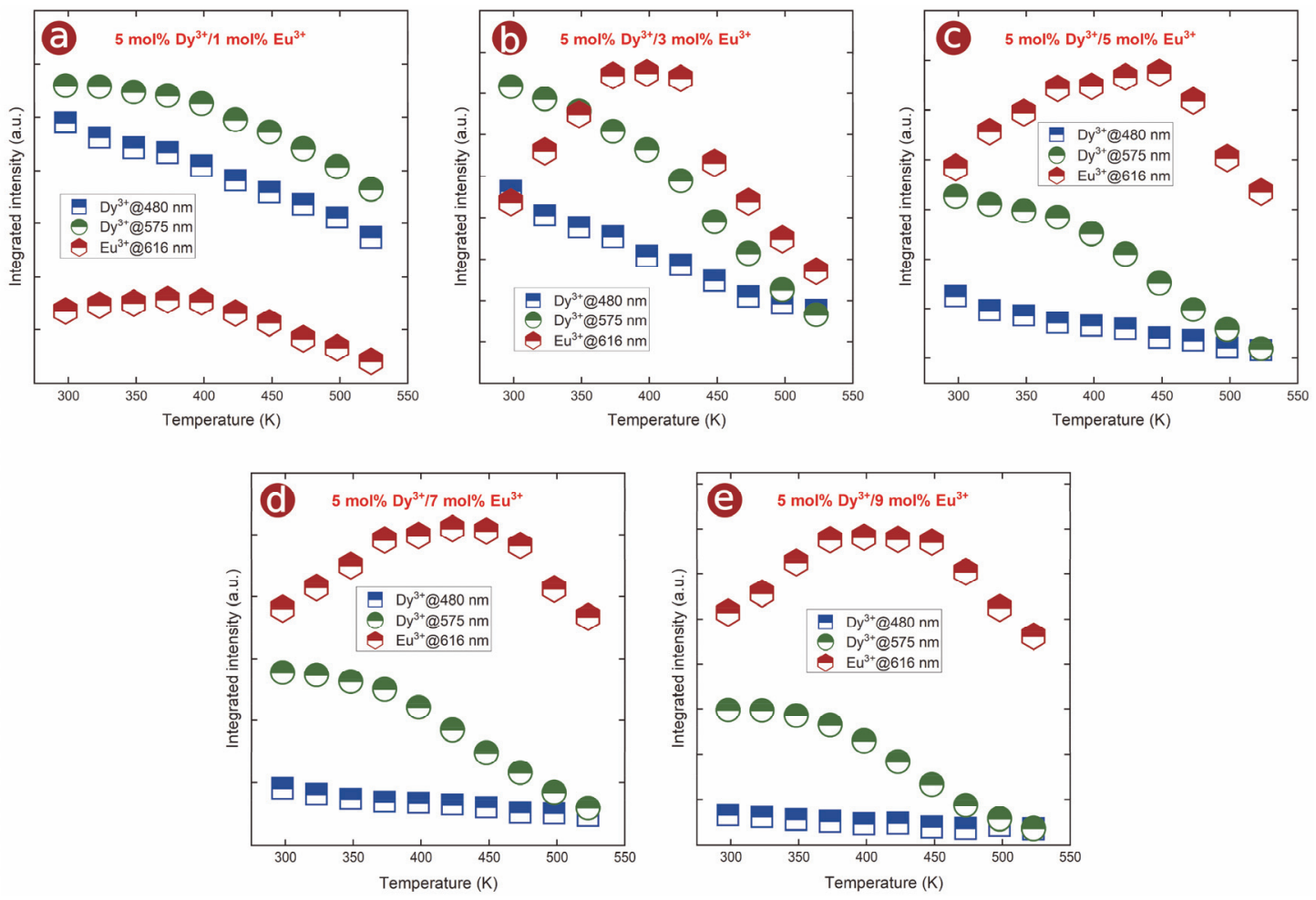

Figure S3. (a)-(d) The dependence of the integrated emission intensity at 480, $575 \mathrm{~nm}$ of $\mathrm{Dy}^{3+}$, and $616 \mathrm{~nm}$ of $\mathrm{Eu}^{3+}$ on temperature for $\mathrm{Dy}^{3+} / \mathrm{Eu}^{3+}$ co-doped $\mathrm{BaLa}_{4} \mathrm{Si}_{3} \mathrm{O}_{13}$ phosphors. 

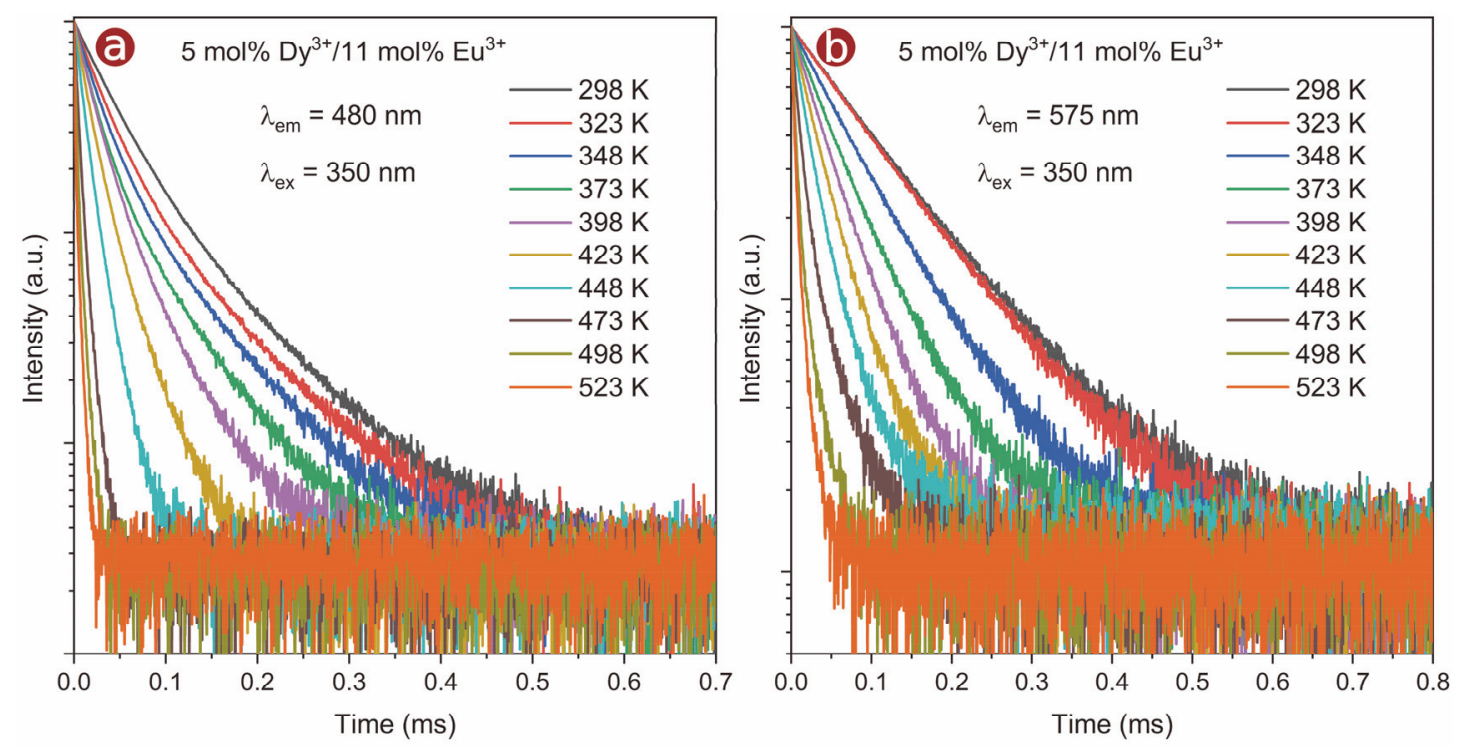

Figure S4. Decay curves monitored at (a) $480 \mathrm{~nm}$, and (b) at $575 \mathrm{~nm}$ in a temperature range from 298 to $523 \mathrm{~K}$ for the $5 \mathrm{~mol} \% \mathrm{Dy}^{3+} / 11 \mathrm{~mol} \% \mathrm{Eu}^{3+}$ co-doped $\mathrm{BaLa}_{4} \mathrm{Si}_{3} \mathrm{O}_{13}$ phosphors. The excitation wavelength is $350 \mathrm{~nm}$. 


\section{REFERENCES}

(1) Li, H.; Zhao, R.; Jia, Y.; Sun, W.; Fu, J.; Jiang, L.; Zhang, S.; Pang, R.; Li, C. $\mathrm{Sr}_{1.7} \mathrm{Zn}_{0 .} \mathrm{CeO}_{4}: \mathrm{Eu}^{3+}$ novel red-emitting phosphors: synthesis and photoluminescence properties. ACS Appl. Mater. Interfaces 2014, 6 (5), 3163-3169.

(2) Ci, Z.; Guan, R.; Nie, K.; Liu, L.; Han, L.; Zhang, J.; Wang, Y. Temperaturesensitive photoluminescence property and energy transfer mechanism in the silicate phosphor $\mathrm{MgY}_{4} \mathrm{Si}_{3} \mathrm{O}_{13}: \mathrm{Eu}^{3+}$. J. Am. Ceram. Soc. 2015, 98 (8), 2488-2492.

(3) Li, B.; Huang, X.; Guo, H.; Zeng, Y. Energy transfer and tunable photoluminescence of LaBWO6: $\mathrm{Tb}^{3+}, \mathrm{Eu}^{3+}$ phosphors for near-UV white LEDs. Dyes Pigm. 2018, 150, 67-72.

(4) Guo, H.; Huang, X.; Zeng, Y. Synthesis and photoluminescence properties of novel highly thermal-stable red-emitting $\mathrm{Na}_{3} \mathrm{Sc}_{2}\left(\mathrm{PO}_{4}\right)_{3}: \mathrm{Eu}^{3+}$ phosphors for UVexcited white-light-emitting diodes. J. Alloys Compd. 2018, 741, 300-306.

(5) Panigrahi, K.; Saha, S.; Sain, S.; Chatterjee, R.; Das, A.; Ghorai, U. K.; Das, N. S.; Chattopadhyay, K. K. White light emitting $\mathrm{MgAl}_{2} \mathrm{O}_{4}$ : $\mathrm{Dy}^{3+}, \mathrm{Eu}^{3+}$ nanophosphor for multifunctional applications. Dalton Trans. 2018, 47 (35), 12228-12242.

(6) Zhou, W.; Song, M.; Zhang, Y.; Xie, Z.; Zhao, W. Color tunable luminescence and optical temperature sensing performance in a single-phased $\mathrm{KBaGd}\left(\mathrm{WO}_{4}\right)_{3}$ : $\mathrm{Dy}^{3+}$, $\mathrm{Eu}^{3+}$ phosphor. Opt. Mater. 2020, 109, 110271.

(7) Wang, J.; Bu, Y.; Wang, X.; Seo, H. J. A novel optical thermometry based on the energy transfer from charge transfer band to $\mathrm{Eu}^{3+}-\mathrm{Dy}^{3+}$ ions. Sci. Rep. 2017, 7 (1), $1-11$.

(8) Suo, H.; Guo, C.; Li, T. Broad-scope thermometry based on dual-color modulation up-conversion phosphor $\mathrm{Ba}_{5} \mathrm{Gd}_{8} \mathrm{Zn}_{4} \mathrm{O}_{21}: \mathrm{Er}^{3+} / \mathrm{Yb}^{3+}$. J. Phys. Chem. C 2016, 120 (5), 2914-2924.

(9) Zhang, X.; Xu, J.; Guo, Z.; Gong, M. Luminescence and energy transfer of dualemitting solid solution phosphors $(\mathrm{Ca}, \mathrm{Sr})_{10} \mathrm{Li}\left(\mathrm{PO}_{4}\right)_{7}: \mathrm{Ce}^{3+}, \mathrm{Mn}^{2+}$ for ratiometric temperature sensing. Ind. Eng. Chem. Res. 2017, 56 (4), 890-898.

(10) Zhang, X.; Huang, Y.; Gong, M. Dual-emitting $\mathrm{Ce}^{3+}, \mathrm{Tb}^{3+}$ co-doped LaOBr phosphor: luminescence, energy transfer and ratiometric temperature sensing. Chem. Eng. J. 2017, 307, 291-299.

(11) Dong, B.; Cao, B.; He, Y.; Liu, Z.; Li, Z.; Feng, Z. Temperature sensing and in vivo imaging by molybdenum sensitized visible upconversion luminescence of rareearth oxides. Adv. Mater. 2012, 24 (15), 1987-1993.

(12) Li, L.; Tang, X.; Wu, Z.; Zheng, Y.; Jiang, S.; Tang, X.; Xiang, G.; Zhou, X. Simultaneously tuning emission color and realizing optical thermometry via efficient $\mathrm{Tb}^{3+} \rightarrow \mathrm{Eu}^{3+}$ energy transfer in whitlockite-type phosphate multifunctional phosphors. J. Alloys Compd. 2019, 780, 266-275.

(13) Ding, M.; Lu, C.; Chen, L.; Ji, Z. $\mathrm{Ce}^{3+} / \mathrm{Tb}^{3+}$ co-doped $\beta-\mathrm{NaYF}_{4}$ dual-emitting phosphors for self-referencing optical thermometry. J. Alloys Compd. 2018, 763, 8593.

(14) Chen, X.; Zheng, Z.; Teng, L.; Wei, R.; Hu, F.; Guo, H. Self-calibrated optical thermometer based on luminescence from $\mathrm{SrLu}_{2} \mathrm{O}_{4}: \mathrm{Bi}^{3+}, \mathrm{Eu}^{3+}$ phosphors. RSC Adv. 2018, 8 (62), 35422-35428. 